\title{
Cuotas de género y acceso femenino al Parlamento: los casos de Argentina y Brasil en perspectiva comparada*
}

\author{
Jutta MARX, Jutta BORNER y \\ Mariana CAMINOTTI
}

\section{Género y políticas de acción afirmativa en América Latina}

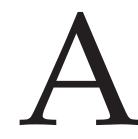

1 calor de los procesos de democratización política, los ciudadanos y ciudadanas de América Latina recuperaron el derecho de elegir a sus representantes y de postularse para desempeñar cargos electivos. En este marco, si bien las mujeres se involucraron activamente en las luchas contra las dictaduras militares y en los movimientos de defensa de los derechos humanos, durante las denominadas transiciones su acceso a las instancias decisorias y representativas fue acentuadamente limitado. A modo ilustrativo conviene señalar que, hacia 1990, la presencia femenina en las Cámaras altas de la región era del orden del 5\%, mientras que en las Cámaras bajas rondaba el 9\% (Barreiro et al., 2004).

Frente a esta realidad -que puso de relieve la permanencia de barreras de naturaleza estructural que dificultan una participación más igualitaria en el ejercicio del poder político- y en medio de un clima internacional favorable a la promoción de una mayor equidad entre los géneros ${ }^{1}$, la década pasada fue el escenario de un conjunto de reivindicaciones y de negociaciones que darían lugar a la adopción de cuotas para candida-

\footnotetext{
* A lo largo del artículo, las traducciones del portugués al español, incluyendo citas bibliográficas y de fragmentos de legislación, son propias.

1. Debe destacarse especialmente el papel desempeñado por Naciones Unidas a partir de la declaración del Año Internacional de la Mujer en 1975 y del inicio de la década (19751985). En 1976 se firmó la Convención contra Todas las Formas de Discriminación de la Mujer (CEDAW) y en 1995 la Plataforma de Acción, firmada en la IV Conferencia Mundial de la Mujer celebrada en Beijing, marcó un hito importante al incorporar el objetivo de alcanzar una mayor igualdad entre los géneros en el ámbito del poder político.
} 
turas a cargos legislativos en una decena de países de la región². Conviene precisar que las cuotas constituyen medidas compensatorias y redistributivas encaminadas a subsanar situaciones de desigualdad a partir de un tratamiento diferenciado, con el propósito de posibilitar la inclusión sistemática de ciertos segmentos o categorías sociales en las instituciones representativas y en los procesos de toma de decisiones.

La experiencia internacional señala que el éxito de las cuotas de género depende en gran medida de las características del sistema electoral, de la precisión de las normas que sustentan este tipo de medidas, y de la activa participación de los actores nacionales en su instrumentación y vigilancia por su correcta aplicación. De hecho, en los últimos años diversos estudios han demostrado que los sistemas de representación proporcional son más favorables que los mixtos y los mayoritarios ${ }^{3}$. Asimismo, el tipo de lista electoral es un factor de suma relevancia ${ }^{4}$.

Las cuotas presentan mayores probabilidades de éxito cuando son aplicadas en el marco de listas cerradas, en las cuales el elector debe votar la nómina completa de un partido sin la posibilidad de efectuar cambios. Lo contrario ocurre en el caso de las listas abiertas, donde los candidatos no están jerárquicamente ordenados, correspondiéndole al elector escoger uno de los nombres presentados por el partido según su preferencia. Aun así, las condiciones de eficacia de las cuotas bajo listas cerradas se ven condicionadas por la existencia de una definición clara que asegure que las candidatas sean emplazadas en lugares expectables (esto es, con probabilidades de resultar elegidas). La precisión en la redacción de las leyes, por su parte, permite conjurar el riesgo de que surjan interpretaciones divergentes que puedan acarrear luchas judiciales para la resolución de los conflictos (como, de hecho, ocurrió inicialmente en el caso argentino). La literatura especializada también ha puesto de relieve la importancia de que se establezca de manera explícita un sistema de sanciones que penalice el incumplimiento de los requisitos previstos en la legislación (Dahlerup y Freidenvall, 2003). A propósito, se observa una

2. En Argentina (30\%, ambas Cámaras), Bolivia (30\% Cámara baja, 25\% Senado), Brasil (30\%, Cámara baja), Costa Rica (40\%, congresistas), República Dominicana (25\%, congresistas), Ecuador $(30 \%$, congresistas), Honduras $(30 \%$, congresistas), México (30\%, ambas cámaras), Panamá (30\%, congresistas), Paraguay (20\%, ambas cámaras), Perú (25\%, congresistas) y Venezuela ( $30 \%$, ambas cámaras) se sancionaron leyes de cuotas para candidaturas a cargos legislativos (IDEA, http: / / www.quotaproject.org/).

3. Entre los trabajos que presentan esta perspectiva, ver Barreiro et al. (2004), Jones y Navia (1999), Matland (2004), Rule (1994) y Reynolds (1999), entre otros.

4. Si bien existen diferentes definiciones respecto de los tipos de listas partidarias, en el marco de este artículo seguimos la clasificación de Nicolau (1999; ver Araújo, 1999), quien establece una distinción entre listas cerradas (donde el partido define la composición y ordena a los candidatos de acuerdo con su prioridad de elección), abiertas (donde el partido presenta una nómina de candidatos sin un ordenamiento jerárquico), flexibles (donde el partido presenta una lista de candidatos jerárquicamente ordenada que los electores pueden alterar o confirmar según sus preferencias), y libres (como la adoptada en Suiza). 
amplia gama de medidas que van desde la aplicación de multas (Francia) hasta el rechazo de listas (Argentina). La aplicación efectiva de las sanciones depende, en muchos casos, del control que ejercen las organizaciones de mujeres y las aspirantes a candidaturas.

A la luz de lo expuesto, este artículo analiza los diferentes resultados alcanzados respecto del propósito de estimular un mayor acceso femenino a los congresos nacionales de Argentina (país internacionalmente considerado como un claro ejemplo de una cuota exitosa) y Brasil (donde las cuotas han mostrado un desempeño menos alentador y más incierto $)^{5}$. Específicamente, se argumentará que la interacción entre las dimensiones arriba señaladas contribuye a comprender estos distintos derroteros y que la comparación arroja algunas enseñanzas para favorecer la implementación de políticas de acción afirmativa tendientes a incrementar la presencia legislativa de mujeres en otros países.

En 1991, con la sanción de la Ley 24.012 -Ley de Cupo Femenino- Argentina se transformó en el primer país que adoptó una cuota mínima (30\%) para candidaturas de mujeres a través de la reforma de su legislación electoral. De este modo, la legislación argentina se diferenció de la experiencia de los países europeos en los cuales se establecieron sistemas de cuotas voluntarias a partir de la reforma de las cartas orgánicas o de las declaraciones de principios de los partidos políticos. En principio, este piso mínimo de participación femenina sólo se aplicó a las listas de candidatos a diputados nacionales. A partir de las elecciones del año 2001 -cuando los senadores nacionales pasaron a ser electos de manera directa- la Ley de Cupo Femenino se extendió a las listas de candidatos a la Cámara alta. Por su parte, en 1995 se sancionó en Brasil una cuota mínima del $20 \%$ para candidaturas femeninas en las elecciones de nivel municipal y, en 1997, una nueva ley electoral estableció que cada partido o coalición debía reservar un mínimo de $30 \%$ y un máximo de $70 \%$ de las candidaturas de cada sexo a todos los cargos electos proporcionalmente (Ley 9.504, Art. 10, inciso 3) ${ }^{6}$. En virtud de una cláusula transitoria, en las elecciones legislativas de 1998 la reserva mínima fue del 25\%.

A partir de la entrada en vigor de la Ley de Cupo Femenino en 1993, en Argentina se produjo un notable incremento de la cantidad de legisladoras nacionales. En Brasil, en cambio, luego de la sanción de las cuotas se

5. Otra modalidad de evaluación de las cuotas de género se centra en el examen de los resultados logrados desde el punto de vista de la representación sustantiva de mujeres y la incorporación de cuestiones de género en la agenda pública. En el marco de una investigación más amplia en curso, analizamos las experiencias de Argentina y Brasil desde ambas perspectivas.

6. De este modo, las cuotas brasileñas comprenden las candidaturas para la Cámara Federal de Diputados, la Cámara Legislativa del Distrito Federal, las Asambleas Legislativas estaduales y las Cámaras Municipales, y no se aplican a la elección de senadores nacionales. 
evidenciaron desarrollos menos alentadores e incluso paradójicos. De hecho, los partidos brasileños no han logrado respetar en ninguna de las elecciones realizadas hasta la fecha los porcentajes legales mínimos exigidos para las candidaturas de cada sexo. Aún más, luego de la primera experiencia electoral con cuotas a nivel federal en el año 1998, el número de diputadas federales disminuyó con respecto a la elección anterior (sin cuotas).

\section{Las trayectorias de las cuotas en Argentina y Brasil}

Más allá de ciertas diferencias significativas en su historia política, durante gran parte del siglo XX las mujeres brasileñas y argentinas se vieron marginadas del pleno disfrute de su ciudadanía política. En Brasil, el derecho al sufragio femenino fue reconocido en 1932 y, en el caso argentino, esta conquista se remonta a 1947. Con la sola excepción del período del segundo gobierno de Perón, en la Argentina de los años cincuenta, y de una leve recuperación durante el tercer gobierno peronista (19731976), la presencia de mujeres en las instituciones representativas ha sido, por lo general, extremadamente limitada en ambos países.

En Brasil, la históricamente exigua presencia femenina en el Poder Legislativo federal experimentó un primer punto de inflexión luego de la recuperación democrática de mediados de los años ochenta. Entre 1983 y 1987, las mujeres pasaron de representar el 1,67\% a conformar el 5,36\% de la Cámara baja, cuando resultaron elegidas 26 diputadas federales. Al año siguiente, cuando el Congreso Nacional se transformó en la Asamblea Constituyente convocada para reformular el marco constitucional del país, las legisladoras actuaron articuladamente entre sí y con distintas organizaciones de mujeres. Aglutinadas tras el eslogan "Viva la diferencia, con derechos iguales", su accionar daría lugar a lo que en ese momento se popularizó con el nombre de lobby do batom (lápiz de labio) ${ }^{7}$. Al mismo tiempo, estas parlamentarias llegaron a elaborar un manifiesto que llamaba la atención sobre la situación de discriminación de las mujeres en la sociedad y sobre su condición de minoría en el ámbito legislativo.

En Argentina, en el marco de la recuperación de la democracia, las mujeres desempeñaron un papel protagónico en los movimientos de defensa de los derechos humanos y, al mismo tiempo, se involucraron masivamente en las bases de los partidos políticos ${ }^{8}$. No obstante, esa participación no se vio reflejada en un acceso igualitario al ejercicio de cargos

7. A partir de entonces, se inauguró una experiencia de coordinación al interior de la Cámara Federal y del Senado que aglutina a las parlamentarias de distintos signos partidarios para el tratamiento de proyectos de género y que se ha dado en llamar bancada femenina.

8. Para el caso de la Unión Cívica Radical, ver Marx (1992). 
electivos. Por el contrario, entre 1983 y 1989 la presencia femenina en el Congreso de la Nación no alcanzó siquiera el 5\% de las bancas y las mujeres tampoco ejercieron cargos de conducción en los partidos políticos mayoritarios (Unión Cívica Radical y Partido Justicialista).

Las leyes de cuotas sancionadas en la década de 1990 significaron un cuestionamiento explícito de esta situación, y fueron diseñadas con el propósito de contribuir a remover los obstáculos que han enfrentado históricamente las mujeres para acceder a la competencia electoral y al desempeño de cargos representativos en condiciones de mayor igualdad. Con el fin de comprender cómo opera cada legislación, es preciso tomar en consideración el modo en que se lleva a cabo la elección de representantes a la Cámara de Diputados y al Senado en cada caso.

\section{Rasgos político institucionales básicos y modalidades de elección legislativa}

Argentina y Brasil son países federales con un diseño bicameral del Poder Legislativo nacional. En ambos casos, el reclutamiento y la nominación de candidatos se desarrolla a nivel de distritos (provincias y estados respectivamente) $)^{9}$.

La Cámara de Diputados de Argentina cuenta con 257 integrantes elegidos en veinticuatro distritos electorales por medio de un sistema de representación proporcional con listas partidarias cerradas ${ }^{10}$. El mandato de los diputados nacionales es de cuatro años, pero la Cámara se renueva cada dos años por mitades. El Senado está integrado por 72 legisladores ( 3 por cada distrito electoral), elegidos mediante un sistema de lista incompleta que asegura dos escaños para el partido que obtiene mayor cantidad de votos y uno para la fuerza subsiguiente (mayoría atenuada). El mandato de los senadores nacionales es de seis años y la Cámara alta se renueva bianualmente por tercios en elecciones escalonadas ${ }^{11}$.

La Cámara Federal de Diputados de Brasil posee 513 miembros elegidos en veintisiete distritos electorales por medio de un sistema de representación proporcional con listas abiertas para un período de cuatro años $^{12}$. Bajo esta modalidad, cada partido o coalición presenta una nó-

9. Para un análisis pormenorizado de ambos diseños institucionales desde una perspectiva comparada, ver Abal Medina et al. (2003).

10. La cantidad de diputados por distrito depende de la población de cada uno de ellos y oscila entre un mínimo de 5 y un máximo de 70 escaños. Sin embargo, como consecuencia de la renovación parcial de la Cámara, las magnitudes efectivas de distrito poseen un rango de 2 a 35 escaños.

11. En este caso, cada dos años un tercio de los distritos renuevan la totalidad de sus bancas en el Senado.

12. Al igual que en Argentina, la magnitud de los distritos varía de acuerdo con la cantidad de población. La Constitución establece, en este caso, un mínimo de 8 y un máximo de 70 escaños por estado. 
mina de candidatos sin un ordenamiento jerárquico y el elector puede optar por emitir su voto a favor de un partido o coalición en su conjunto (voto "de legenda") o de un candidato individual (voto preferencial o uninominal), que de hecho prevalece ampliamente en la práctica. La suma de los votos "de legenda" y uninominales determina la cantidad de escaños que recibirá un partido o coalición. Las bancas se adjudican en base al voto preferencial, de modo tal que resultarán electos aquellos candidatos que hayan obtenido mayor cantidad de votos uninominales (Nohlen, 1995).

Finalmente, el Senado brasileño cuenta con 81 legisladores (3 por cada distrito electoral) electos por un sistema mayoritario para un período de ocho años. Dado que la renovación de la Cámara alta es parcial, se realizan elecciones uninominales por mayoría simple cuando se renueva un tercio de la misma (un senador por distrito), y binominales cuando se renuevan los dos tercios restantes (Abal Medina et al., 2003)13. En virtud de que las cuotas de género adoptadas en Brasil comprenden sólo a los cargos electos proporcionalmente, éstas no se aplican a la elección de senadores.

\section{Orígenes y características de las legislaciones adoptadas}

En Argentina, la sanción de la Ley de Cupo Femenino en 1991 fue el resultado de una lucha protagonizada por las mujeres políticas a partir de fines de los años ochenta, acompañada por sectores del feminismo y del movimiento de mujeres. Inicialmente, observando el ejemplo de los países europeos, se ensayaron tentativas de negociación con el propósito de impulsar la introducción de cuotas en las cartas orgánicas partidarias. Ante la reacción negativa de los partidos políticos mayoritarios, el fracaso al que parecía conducir esta estrategia fue un factor relevante para el desarrollo de dos proyectos de ley presentados en el mes de noviembre de 1989, uno en cada Cámara del Congreso de la Nación, que propusieron reformar el Código Electoral Nacional con el objetivo de obligar a todas las organizaciones partidarias a incluir más mujeres en las listas de candidatos a cargos legislativos.

El primero de ellos -redactado por la senadora nacional por la provincia de Mendoza, Margarita Malharro de Torres (UCR), como consecuencia de un debate entablado en el Congreso Nacional de Mujeres Radicales en el año 1989- preveía la obligación de que los partidos políticos incluyeran un mínimo de $30 \%$ de mujeres en sus listas de candidatos en aquellas posiciones que les dieran posibilidades de resultar electas. En el

13. En los distritos uninominales (renovación de un tercio del Senado) decide la mayoría relativa de los votos. En los binominales (renovación de dos tercios) los escaños son distribuidos entre los dos aspirantes que reciban la mayor cantidad de sufragios. 
marco del proyecto presentado en la Cámara baja -en este caso por un grupo multipartidario de diputadas- la redacción era neutra con relación al género, pero se preveían exigencias claras para la ubicación de los candidatos de cada sexo en las listas. En particular, esta propuesta estipulaba que las listas partidarias para cargos legislativos no podían incluir más del $70 \%$ de personas del mismo sexo y que, por cada dos candidatos de un sexo, debía ubicarse uno del otro.

Con el propósito de apoyar y promover ambas propuestas, las mujeres desarrollaron múltiples iniciativas que gravitaron de forma decisiva en la posterior sanción de la Ley de Cupo Femenino. En 1990 se conformó la Red de Feministas Políticas, integrada por mujeres de quince organizaciones partidarias. Bajo la consigna Con pocas mujeres en politica, cambian las mujeres; con muchas mujeres en política, cambia la política, esta alianza se constituyó en un actor protagónico en el contexto de la discusión y aprobación de las cuotas (Archenti, 2000). Paralelamente, el $5^{\circ}$ Encuentro Nacional de Mujeres ${ }^{14}$, que reunió una amplia gama de organizaciones y activistas en la localidad Termas de Río Hondo (provincia de Córdoba), expresó su apoyo explícito en esta dirección y, como medida práctica, “aprobó el envío de notas a los presidentes de todos los partidos políticos y de ambas Cámaras para la aprobación de los dos proyectos presentados" (Consejo Nacional de la Mujer, 1994: 45). Con el apoyo del Consejo Nacional de la Mujer $(\mathrm{CNM})^{15}$ se coordinaron manifestaciones y acciones de difusión de los proyectos de ley en todo el país.

El presidente Carlos S. Menem (1989-1999) hizo propia la demanda por la introducción de acciones afirmativas, una medida coherente con su política de "modernizar" el país. No obstante, el apoyo presidencial a la Ley de Cupo Femenino se debió, además, a la necesidad de dar respuesta a los reclamos planteados por las mujeres de su propio partido, el Partido Justicialista (Barrancos, 2002). Este respaldo, la persistencia de las mujeres de una gran parte de los partidos políticos (especialmente de la UCR y el PJ), el apoyo de otras organizaciones como la Multisectorial de la Mujer -que nucleaba grupos feministas, militantes de partidos políticos e integrantes de organizaciones de la sociedad civil- y el accionar del CNM, fueron cruciales para lograr la aprobación de la norma (Gray, 2003).

Con la sanción de la Ley 24.012 -basada en la propuesta de la senadora Malharro de Torres- el 29 de noviembre de 1991, el artículo 60 del Código Electoral quedó reformado con la siguiente especificación:

14. El primer Encuentro se realizó en el año 1986 y se siguen llevando a cabo anualmente. 15. Bajo el gobierno de Raúl Alfonsín se creó la Dirección Nacional de la Mujer dependiente de la Secretaría de Desarrollo Humano y Familia del Ministerio de Salud y Acción Social, que en 1987 fue transformada en la Subsecretaría de la Mujer. En 1992, ya bajo el gobierno de Carlos Menem, la Subsecretaría se constituyó en el Consejo Nacional de la Mujer (CNM), con dependencia directa de la Presidencia de la Nación y, a partir de la reforma constitucional de 1994, de la Jefatura de Gabinete de Ministros. 
Las listas que se presenten deberán tener mujeres en un mínimo de un treinta por ciento $(30 \%)$ de los candidatos de los cargos a elegir y en proporciones con posibilidad de resultar electas. No será oficializada ninguna lista que no cumpla estos requisitos.

El 8 de marzo de 1993 fue promulgado el decreto $N^{\circ} 379$, reglamentario de la ley, que definió más explícitamente el mecanismo del cupo femenino. Este decreto estableció que "la finalidad de la Ley es lograr la integración efectiva de la mujer en la actividad política, evitando su postergación al no incluirse candidatos femeninos entre los candidatos con expectativa de resultar electos". Con este fin, se especificó que el cupo del 30\% debía entenderse como la cantidad mínima y que debía incluirse una mujer por cada dos varones. En el caso de que se renovaran solamente dos cargos, al menos uno de ellos debía tener como candidata a una mujer.

A pesar de la relativa claridad de la norma con respecto al emplazamiento de las candidatas en las listas partidarias, en las elecciones legislativas de 1993 "los partidos políticos presentaron sus listas con un 'criterio minimalista', es decir, con un 30\% de mujeres en su composición pero ocupando éstas los últimos lugares" (Tula, 2002: 67). Para ello, se basaron en el argumento de que la disposición de incluir una mujer como mínimo en el segundo lugar de la lista si un partido no renovaba más de dos bancas significaba una alteración del espíritu de la ley, dado que transformaría el cupo del 30\% en uno del 50\% (Lázzaro y Fraquelli, 2003). Desde esta perspectiva, interpretaron que la expresión "con probabilidad de ser electa" debía referirse a la totalidad de los escaños a renovar en una provincia y no a los de un partido, puesto que, hipotéticamente, cualquier partido podría ganar la totalidad de las bancas en juego.

Estas circunstancias originaron nuevamente una notable solidaridad entre las mujeres de diversas fuerzas políticas que habían luchado por la sanción de la Ley de Cupo Femenino, quienes se organizaron para velar por su cumplimiento. Un obstáculo que se presentó en este contexto estuvo dado por las restricciones legales que dificultaron la impugnación de listas que no cumplían con los requisitos mínimos establecidos. Ello se debió, sobre todo, a la negativa de la justicia electoral de aceptar pleitos que no fueran presentados por un "particular damnificado", es decir, por una candidata. De este modo, las candidatas que tenían que afrontar por sí mismas la presentación judicial se veían sometidas a fuertes presiones por parte de sus organizaciones partidarias (Tula, 2002).

A pesar de la presión sufrida y del peligro de recibir sanciones partidarias, varias candidatas se presentaron ante la justicia y lograron los primeros fallos en su favor. En uno de ellos, de particular importancia, la Cámara Nacional Electoral (CNE) ordenó la ubicación de la candidata Darci Beatriz Sampietro, de la UCR de Entre Ríos, en el tercer lugar de la lista. En su extensa fundamentación -más tarde confirmada por la Corte 
Suprema de Justicia de la Nación- la CNE aclaró que "la interpretación de la Ley 24.012/91 y su decreto reglamentario 379/93 debía hacerse con arreglo a la realidad, descartándose así la mera posibilidad de que todo candidato sea potencialmente susceptible de ser electo" (Tula, 2002: 70). Es decir, la referencia debía ser el número de bancas a renovar de cada partido y no la totalidad de los escaños que corresponden a un distrito electoral.

Como consecuencia de la incorporación del artículo 37 sobre la igualdad de oportunidades para varones y mujeres en la Constitución Nacional reformada en 1994, la CNE reconoció en 1995 al Consejo Nacional de la Mujer la legitimidad para iniciar acciones legales en defensa de las candidatas, posibilitando de ese modo la actuación judicial del organismo. Ese mismo año, el Consejo patrocinó varias causas para lograr el cumplimiento del cupo femenino. En este contexto, entre 1993 y 2003 se contabilizó un total de 1.273 resoluciones de la Cámara Nacional Electoral sobre la materia (Lázzaro y Fraquelli, 2003).

Otro factor importante para lograr el efectivo cumplimiento de la Ley de Cupo Femenino fue, sin duda, la intervención de la Comisión Interamericana de Derechos Humanos (CIDH) a favor de Maria Merciadri de Morini, afiliada de la UCR, quien en 1994 había efectuado una denuncia ante esa instancia por "la violación de los derechos al debido proceso, a los derechos políticos, a la igualdad ante la ley y a los recursos efectivos por parte de la República Argentina en su perjuicio" (idem, 2003: 9). La $\mathrm{CIDH}$ admitió el caso e intervino frente al gobierno del presidente Fernando de la Rúa, quien había triunfado en las elecciones de octubre de 1999 como candidato de la Alianza UCR-FREPASO, sucediendo a Carlos Menem en la Presidencia de la República. El 8 de marzo de 2001, se llegó a una solución amistosa del conflicto.

En síntesis, las demandas judiciales iniciadas por mujeres políticas cuyas acciones trascendieron las fronteras partidarias obligaron a los partidos políticos a cumplir con la norma y evitaron que, en la práctica, se desvirtuara el alcance de la Ley de Cupo Femenino. Como consecuencia de estos conflictos, el 28 de diciembre de 2000 el presidente De la Rúa promulgó un nuevo decreto reglamentario ( $\left.\mathrm{N}^{\mathrm{o}} 1246\right)$ que sustituyó al vigente hasta entonces. En este marco, se estipularía que: i) la cuota electoral se aplica a todos los cargos electivos de diputados, senadores y constituyentes nacionales; ii) el $30 \%$ de las candidaturas que deben ser destinadas a mujeres se refiere a la cantidad mínima; iii) la cuota sólo se considera cumplida cuando es aplicada al número de cargos que cada organización partidaria renueva en la elección correspondiente. Además, se estableció una casuística extensa para determinar la interpretación del cupo femenino en diversos casos que pudieran dar lugar a duda.

Por otra parte, el nuevo decreto reglamentario definió explícitamente las sanciones aplicables a las listas que no cumplan con la norma. De este 
modo quedó establecido que si el juez con competencia electoral determina que alguna de las candidatas que integran el mínimo del 30\% estipulado en la Ley 24.012 no reúne las cualidades exigidas para el cargo al cual se postula, o que está ubicada en un lugar posterior que el que le corresponde en la lista, el partido en cuestión posee un plazo de 48 horas para proceder a su sustitución o reubicación. En caso contrario, el Tribunal ubicará de oficio a las mujeres que siguen en la lista en los lugares correspondientes. Otro punto digno de mención es la disposición según la cual todas las personas inscriptas en el padrón electoral de un distrito tienen el derecho de impugnar una lista si consideran que ésta viola la Ley 24.012. En virtud del conjunto de las disposiciones señaladas, a partir de la entrada en vigor del decreto 1246/00 se redujeron severamente las posibilidades de incumplir las exigencias estipuladas por la Ley de Cupo Femenino.

A diferencia de lo observado en Argentina, en el caso de Brasil la adopción de cuotas no fue el corolario de una estrategia de lucha colectiva ni de alianzas entabladas entre las parlamentarias y sectores del movimiento de mujeres. De acuerdo con algunas interpretaciones, en los inicios de los años noventa la atención de los legisladores brasileños estaba fundamentalmente concentrada en la reglamentación de la Constitución sancionada en 1988. La promoción de una mayor presencia femenina en el Poder Legislativo mediante mecanismos de acción afirmativa no constituía entonces una preocupación central de las mujeres políticas (Miguel, 2000). Sin embargo, Brasil poseía antecedentes interesantes en los ámbitos sindical y partidario.

En 1991, el Partido de los Trabajadores (PT) adoptó una cuota mínima del $30 \%$ para cualquiera de los sexos en sus órganos de dirección, transformándose en uno de los primeros partidos políticos latinoamericanos que promovieron una mayor igualdad entre varones y mujeres por esta vía (Htun y Jones, 2002). Dos años después, la Central Única de Trabajadores (CUT) estableció un porcentaje mínimo de 30\% y un máximo de $70 \%$ para cada sexo en sus instancias de dirección de nivel nacional, estadual y regional, y en 1997 una convención nacional del Partido Democrático Trabalhista (PDT) instituyó una cuota mínima del 20\% de mujeres para sus instancias directivas. Siguiendo a Araújo (1999), sin embargo, ningún partido brasileño adoptó cuotas de género en sus listas de candidatos para escaños legislativos de forma voluntaria, y tampoco hay registros que señalen la discusión de este tipo de medidas en otros sectores $\mathrm{u}$ organizaciones sociales.

En 1995, la iniciativa de establecer una cuota mínima para candidaturas femeninas fue ideada por la entonces diputada federal Marta Suplicy (PT/SP) luego de participar en un encuentro de legisladoras latinoamericanas realizado en San Pablo en el marco del PARLATINO, como parte de las acciones preparatorias para la IV Conferencia Internacional de la Mujer que se realizaría ese mismo año en Beijing. En esa oportunidad, se 
discutió la reciente aprobación de la Ley de Cupo Femenino en Argentina, así como los sistemas de cuotas adoptados en otras experiencias internacionales (Htun y Jones, 2002).

El proyecto de ley impulsado por Suplicy ( $\left.\mathrm{N}^{\mathrm{0}} 783 / 95\right)$ contó con la firma de más de veinte diputadas de distintos partidos políticos y fue presentado el 10 de agosto de 1995 en la Cámara Federal de Diputados, en un momento en que se discutía la ley electoral que reglamentaría las elecciones municipales de 1996. De este modo, en el mes de septiembre:

[...] paralelamente al projecto [de ley] ya encaminado, se presentó una enmienda a la propuesta de Ley Electoral en discusión. Los diputados Marta Suplicy y Paulo Bernardo propusieron una cuota mínima de 30\% de mujeres a ser incorporada en las listas de candidatos de los partidos (Araújo, 1999: 5).

Un aspecto importante a destacar es que, además de la reserva de un porcentaje mínimo de vacantes para candidaturas de mujeres en las listas partidarias, la propuesta incluía otras medidas específicas de apoyo a las mujeres, especialmente relevantes en el marco del sistema electoral de Brasil, tales como la referencia obligatoria al sexo del candidato en las nóminas electorales ${ }^{16}$, la emisión de mensajes en la televisión y en la propaganda electoral que divulgaran la existencia de las cuotas, y la asignación específica de una porción de los recursos partidarios para el estímulo de las candidaturas femeninas (Araújo, 1999). Con todo, la Ley 9.100, sancionada el 29 de septiembre de 1995, estableció simplemente que "cada partido o coalición podrá registrar candidatos para la Cámara Municipal hasta el ciento veinte por ciento del número de lugares a completar" (artículo 11) y que "[el] veinte por ciento, como mínimo, de las vacantes de cada partido o coalición deberán ser cubiertas por candidaturas de mujeres" (inciso 3).

Como se podrá apreciar, la Ley 9.100/95 no sólo no incorporó las iniciativas adicionales destinadas a paliar la situación de desventaja de las mujeres en el marco de la competencia electoral, sino que también redujo el porcentaje mínimo de $30 \%$ originalmente planteado. Al mismo tiempo, habilitó un aumento del número total de candidatos que los partidos podían presentar (pasando del 100\% al 120\% de las bancas en juego), lo cual permite entrever que la conquista de una primera legislación electoral que aseguró una cuota mínima para candidaturas femeninas no estuvo exenta de negociaciones. A propósito, la misma Suplicy reconocería que "...la propuesta aprobada diluyó la posibilidad de que la cuota contribuya para determinar una mayor inversión [de los partidos] en las campañas de las mujeres candidatas" (Araújo, 1999: 5) y que, en reali-

16. Este aspecto se relaciona con la posibilidad de que los candidatos brasileños a cargos electivos se postulen con sus nombres de pila o con apodos con los cuales son conocidos, los que no siempre indican con precisión el sexo. 
dad, "la reivindicación de las mujeres fue manipulada para aumentar el número de candidaturas" (Miguel, 2000: 42).

Conviene señalar que, aunque esta iniciativa sobre cuotas contó con el apoyo de la bancada femenina en el Congreso Nacional y también de algunas organizaciones no gubernamentales que participaron en las audiencias públicas celebradas en la Cámara de Diputados y en el Senado, al parecer no habría recibido un apoyo sustantivo por parte del movimiento de mujeres. Araújo (1999) menciona, de modo ilustrativo, que en la reunión preparatoria para la Conferencia de Beijing organizada por la Articulação de Mulheres Brasileiras en el mes de junio de 1995, la cuestión de las cuotas fue oportunamente discutida pero no se incluyó ninguna propuesta relativa a la misma en el documento final del encuentro.

En 1997, un año después de las primeras elecciones con una cuota mínima para candidaturas de mujeres -que, como hemos visto, sólo rigió para el nivel municipal- se sancionó una nueva Ley Electoral ( $\mathrm{N}^{\circ}$ 9.540) que pasó a reglamentar todos los comicios posteriores. En su artículo $10^{\circ}$, esta norma estableció que "cada partido podrá registrar candidatos para la Cámara de Diputados, Cámara Legislativa, Asambleas Legislativas y Cámaras Municipales, hasta un ciento cincuenta por ciento del número de los lugares a cubrir"; aclarando, en el inciso $3^{\circ}$, que "del número de vacantes resultantes de las reglas previstas en este artículo, cada partido o coalición deberá reservar un mínimo de treinta por ciento y un máximo de setenta por ciento para candidaturas de cada sexo". Es importante precisar que la exigencia de "reservar" un porcentaje determinado de candidaturas no implica destinarlo necesariamente. Lo que la norma brasileña indica es que si las vacantes para candidaturas de un determinado sexo no se completan deben quedar ociosas (esto es, no pueden completarse con candidatos del otro sexo).

La nueva legislación posee así dos diferencias importantes con respecto a la anterior ( $\mathrm{N}^{\circ}$ 9.100/95). En primer lugar, ya no instituye una cuota mínima para candidaturas de mujeres, sino un sistema de cuotas mínimas y máximas para las candidaturas de cada sexo. En segundo lugar, amplía su ámbito de aplicación a todos los cargos electos de acuerdo con las reglas de la representación proporcional. Al mismo tiempo, el número total de candidatos que cada partido se encuentra habilitado a presentar fue elevado desde un 120\% (1995) hasta el 150\% de los escaños en juego en una votación, aunque no es obligatorio completar ese porcentaje máximo.

Tal como argumenta Htun (2003), el aumento porcentual de las candidaturas puede ser entendido como una suerte de "cláusula de escape" de la ley de cuotas brasileña. Por ejemplo, si en un distrito corresponde elegir diez representantes para la Cámara Federal de Diputados, cada partido puede postular un máximo de quince candidatos. En ese caso, la ley de cuotas requiere que cada partido reserve cuatro de esos lugares para candidaturas de un determinado sexo, pero si un partido no desea- 
ra postular mujeres podría presentar sólo once candidatos varones sin contrariar la ley (idem, 2003).

La presencia de mujeres en el Poder Legislativo a partir de la entrada en vigor de las cuotas

A partir de la aplicación de la Ley de Cupo Femenino en las elecciones legislativas de 1993, en Argentina se produjo un notable aumento de la cantidad de diputadas nacionales. En esa oportunidad, la participación de las legisladoras en la Cámara baja ascendió del 5,4\% al 13,6\%17, para llegar a conformar el 33,5\% del total de diputados en el período 20032005 y el 35,2\% a partir de ese último año, ubicando al país en el noveno puesto del ranking que registra la presencia de mujeres en los parlamentos nacionales del mundo (International Parliamentary Union, marzo de 2006) ${ }^{18}$. En el caso del Senado de la Nación, desde el año 2001 (la primera elección con arreglo al cupo femenino) la participación de las mujeres se sextuplicó. Las senadoras, que en el período 1998/2001 constituían el $6 \%$ de la Cámara alta, pasaron a representar el 36\% de los integrantes del Senado en el período 2001/2003 y el 42\% en 2005.

Esta evolución podría sugerir que, en la experiencia argentina, se logró sortear el riesgo de que las cuotas femeninas, en lugar de constituir un porcentaje mínimo, se transformen en un máximo creando de ese modo un "techo de cristal" para la participación de mujeres en las legislaturas. De ser así, la cantidad y el emplazamiento de las candidatas a diputadas y senadoras nacionales en las listas partidarias deberían superar los requisitos mínimos exigidos en la legislación.

Para determinar si realmente se han producido transformaciones en las prácticas de los partidos respecto de la nominación de candidatas, debería realizarse un análisis de las listas partidarias a nivel de cada distrito, dado que -como consecuencia de la renovación parcial de ambas Cámaras y de otros factores como el relativamente frecuente reemplazo de titulares masculinos por suplentes femeninos-, la composición por sexo del Congreso de la Nación no refleja los resultados de cada elección de manera directa. Si bien ello excede el alcance de este artículo, presentaremos algunos indicadores que iluminan el problema. Uno de ellos, de orden general, viene dado por el número y el porcentaje de las candidatas electas sobre la totalidad de senadores y diputados electos en los comicios legislativos nacionales realizados entre 1993 y 2005, que puede apreciarse en la siguiente tabla.

17. Para comprender este primer resultado logrado a partir de la sanción de la Ley de Cupo Femenino es preciso recordar que la Cámara de Diputados de la Nación se renueva parcialmente, por lo cual en 1993 el cupo sólo tuvo efecto para la renovación de la mitad de las bancas.

18. Ver www.ipu.org. 
Tabla 1

Evolución de la elección de diputadas y senadoras nacionales en Argentina (1993-2005)

\begin{tabular}{|c|c|c|c|c|c|c|c|}
\hline & 1993 & 1995 & 1997 & 1999 & 2001 & 2003 & 2005 \\
\hline \multicolumn{8}{|c|}{ Cámara de Diputados } \\
\hline Mujeres electas & 27 & 35 & 35 & 33 & 41 & 45 & 47 \\
\hline Total electos & 127 & 130 & 127 & 130 & 127 & 130 & 127 \\
\hline$\%$ mujeres & 21,3 & 26,9 & 27,6 & 25,4 & 32,3 & 34,6 & 37,0 \\
\hline \multicolumn{8}{|c|}{ Senado } \\
\hline Mujeres electas & & & & & 26 & 8 & 9 \\
\hline Total electos & & & & & 72 & 24 & 24 \\
\hline$\%$ mujeres & & & & & 36,1 & 33,3 & 37,5 \\
\hline
\end{tabular}

Elaboración propia en base a las nóminas de senadores y diputados nacionales publicadas por el Ministerio del Interior de la Nación.

En el caso del Senado, la proporción de mujeres electas entre 2001 y 2005 no permite presumir una superación de los requisitos mínimos establecidos por la Ley de Cupo Femenino ${ }^{19}$. Los resultados logrados en ambas oportunidades, que superan el piso de un tercio, se deben a que en las dos elecciones mencionadas, en un solo distrito la lista que logró una banca tuvo a una mujer en el primer lugar. En este marco, el hecho de que las senadoras argentinas constituyan el $42 \%$ de la Cámara alta a partir del año 2005 se debe a la situación señalada y a una serie de reemplazos, y no a un "sobrecumplimiento" del cupo.

En cuanto a las diputadas electas desde 1993 se observa -con la excepción del año 1999- un sostenido aumento de su número y de su porcentaje sobre el total de los electos. A pesar de que también aquí la gran mayoría de los partidos políticos no hizo más que cumplir con la legislación, en algunos casos las mujeres lograron mejores ubicaciones de lo estrictamente estipulado. El análisis de las nóminas de diputados electos

19. Cabe recordar que, dado que en cada distrito electoral se eligen tres senadores por medio de una lista incompleta que otorga dos bancas al partido que obtiene mayor cantidad de votos y la restante al subsiguiente, ningún partido puede renovar más de dos escaños y debe postular como mínimo una mujer. En la práctica, esta provisión legal se traduce en un piso efectivo del 33,3\% de senadoras por distrito. Como tendencia general se observa que, en cada distrito, la fuerza mayoritaria envía a la Cámara alta un senador y una senadora, respectivamente, mientras que la minoritaria generalmente es representada por un varón. 
por distrito en los comicios legislativos de 2001 y 2005 publicadas por el Ministerio del Interior permite apreciar que, en ese primer año, el $21 \%$ de las listas partidarias que obtuvieron escaños en la Cámara baja superaron las exigencias mínimas establecidas respecto de la cantidad y el emplazamiento de las candidatas. En 2005, por su parte, las mujeres fueron postuladas en posiciones más favorables en el $29 \%$ de las listas que obtuvieron bancas. Sería interesante observar si esta tendencia se mantiene en el tiempo para poder evaluar si la Ley de Cupo Femenino está estimulando transformaciones en las relaciones entre los géneros al interior de los partidos políticos.

En Brasil -donde las cuotas se aplican en un marco de listas abiertas que no permiten establecer ninguna provisión respecto del emplazamiento de los candidatos- se observan resultados muy distintos a los alcanzados en la experiencia argentina. En 1998 (la primera experiencia electoral con cuotas a nivel federal) la cantidad de diputadas nacionales electas disminuyó con respecto a las elecciones anteriores a la sanción de la Ley 9.504 (1994, sin cuotas). En 2002, a pesar del crecimiento del número de electas, la presencia femenina en la Cámara Federal de Diputados continuó siendo proporcionalmente reducida. Además, como se podrá apreciar en la tabla siguiente, en ambas elecciones la proporción de candidaturas de mujeres resultó inferior a los porcentajes mínimos establecidos en cada caso $(25 \%$ y $30 \%$, respectivamente) por la legislación.

Tabla 2

\section{Candidatos y legisladores electos para la Cámara Federal de Diputados de Brasil, según sexo}

(1994-2002)

\begin{tabular}{|c|c|c|c|c|c|c|c|c|c|}
\hline & \multicolumn{3}{|c|}{1994 Sin cuota } & \multicolumn{3}{|c|}{1998 Cuota mínima $=25 \%$} & \multicolumn{3}{|c|}{2002 Cuota mínima $=30 \%$} \\
\hline & \multirow{2}{*}{$\begin{array}{c}\text { Total } \\
\mathbf{N}^{\circ}\end{array}$} & \multicolumn{2}{|c|}{ Mujeres } & \multirow{2}{*}{$\begin{array}{c}\text { Total } \\
\mathrm{N}^{\circ}\end{array}$} & \multicolumn{2}{|c|}{ Mujeres } & \multirow{2}{*}{$\begin{array}{c}\text { Total } \\
\mathbf{N}^{\circ}\end{array}$} & \multicolumn{2}{|c|}{ Mujeres } \\
\hline & & $\mathbf{N}^{\circ}$ & $\%$ & & $\mathbf{N}^{\circ}$ & $\%$ & & $\mathbf{N}^{\circ}$ & $\%$ \\
\hline Candidatos/as & 2.968 & 184 & 6,2 & 3.451 & 359 & 10,4 & 4.210 & 480 & 11,4 \\
\hline Electos / as & 513 & 32 & 6,2 & 513 & 29 & 5,7 & 513 & 42 & 8,2 \\
\hline
\end{tabular}

Elaboración propia en base a Miguel (2004).

Los datos presentados evidencian la debilidad del sistema de cuotas adoptado en Brasil. En particular, en el marco de un sistema electoral en el cual los cargos de elección proporcional se dirimen a través de listas abiertas que personalizan la contienda electoral e incentivan la competencia intrapartidaria, interviene una serie de factores adicionales que deben ser atendidos si se pretende aumentar de manera expresiva la participación femenina en el Poder Legislativo. A propósito, un hecho 
elemental a señalar es que, si bien los partidos disponen de recursos públicos que conforman los denominados fondos partidarios, éstos resultan insignificantes en relación con el costo real de las campañas. En este contexto, la dimensión del país, el carácter individualizado de la competencia y la escasez de financiamiento público (Araújo, 2003) hacen que las candidatas necesiten contar con recursos propios, ya sea financieros, acceso a los medios masivos de comunicación, contactos personales o grupos de apoyo.

De hecho, un argumento frecuentemente esgrimido en el escenario político brasileño es que no hay suficientes mujeres dispuestas a asumir una candidatura, apreciación compartida por buena parte de las legisladoras de ese país entrevistadas en el año $2005^{20}$. Desde esta perspectiva, lo verdaderamente relevante no sería lograr una postulación (como en el caso argentino), sino estar en condiciones de competir con posibilidades reales de triunfar, con lo cual la reticencia de algunas mujeres a postularse como candidatas respondería a un cálculo racional.

De cara a estas dificultades, desde la sanción de la Ley 9.504 hasta la actualidad en el Congreso brasileño se formularon diversas propuestas destinadas a complementar y adaptar el sistema de cuotas a las singularidades de la competencia electoral del país. Entre las iniciativas presentadas podemos señalar, por su importancia, la disposición de destinar una parte del horario gratuito de campaña en las emisoras de radio y de televisión para la divulgación de la existencia de cuotas en el período que antecede a la nominación de candidatos (PL 418/99, Iara Bernardi y otros 25 parlamentarios) ${ }^{21}$; la destinación del 30\% de los recursos del fondo partidario a la creación de programas de promoción de la participación política de las mujeres, una porción análoga de los recursos financieros de las campañas y del tiempo de propaganda electoral en la radio y la televisión (PL 6216/02, Luiza Erudina); así como otros proyectos que promueven el financiamiento público exclusivo de las campañas electorales y la introducción de listas partidarias cerradas en el marco de la discusión sobre reforma política (originada en el proyecto de ley 5268 de 2001) entablada en el Congreso Nacional de Brasil.

20. En el marco del proyecto Género y Política en el Mercosur, actualmente en realización, entrevistamos al 25\% de las legisladoras nacionales (diputadas y senadoras) de Argentina y Brasil.

21. Para apreciar la relevancia de esta iniciativa conviene tomar en consideración que la ley electoral brasileña no permite la compra de espacio en los medios electrónicos de comunicación y establece la concentración de la propaganda electoral en el denominado horario electoral gratuito destinado a los partidos. 


\section{Síntesis de las experiencias en perspectiva comparada}

Las mujeres de Argentina y Brasil comparten con sus pares de otros países una trayectoria de exclusión y marginación de la arena políticoinstitucional. Durante varias décadas, aún después de haber conquistado el ejercicio de la ciudadanía política, no lograron formar parte de las instituciones representativas 22 . Sólo a partir de los procesos de recuperación democrática de la década de 1980, que pusieron término a las últimas dictaduras militares, Argentina y Brasil se sumaron a aquellos países en los cuales las mujeres comenzaron a reclamar condiciones que les permitieran atenuar sus desventajas en la competencia electoral.

En América Latina, Argentina fue el país líder en ese proceso al sancionar cuotas de género por medio de la reforma de su legislación electoral en 1991. En Brasil, si bien entre 1991 y 1993 el Partido de los Trabajadores y la Central Única de Trabajadores habían adoptado cuotas para cada uno de los sexos en sus órganos de dirección, la primera medida similar para las candidaturas femeninas a cargos electivos se sancionó en 1995. Es interesante observar que, en este caso, las experiencias de referencia para la legitimación de los reclamos de las mujeres políticas y de los modelos a seguir ya no fueron sólo las europeas, sino, en mayor medida, las de países vecinos de la región.

En virtud de estos distintos derroteros, actualmente Argentina y Brasil cuentan con cuotas legales destinadas a obligar al conjunto de los partidos políticos a cumplir con un cierto equilibrio entre los sexos a la hora de confeccionar sus listas para determinados cargos representativos. Sin embargo, si se comparan ambas legislaciones nacionales y sus marcos de aplicación, pueden constatarse considerables diferencias entre ellas.

En primer lugar, la ley electoral argentina prevé un cupo femenino mínimo del 30\% mientras que la norma brasileña, al reservar un porcentaje mínimo del $30 \%$ y un máximo del $70 \%$ para cada uno de los sexos, introduce cuotas neutrales. Aunque la experiencia internacional comparada no permite identificar a priori la existencia de ventajas de un sistema con relación al otro, las cuotas femeninas implican un reconocimiento explícito de la necesidad de superar el déficit estructural que sufren las mujeres en la competencia electoral. Las cuotas neutrales, en cambio, no evidencian las desigualdades existentes en la distribución real del poder entre los géneros.

En segundo lugar, la Ley de Cupo argentina y su primer decreto reglamentario establecieron explícitamente que los lugares en las listas de

22. Como hemos señalado, esta regla sólo se quebró por un breve intervalo en la Argentina durante el segundo gobierno de Perón y el tercer gobierno peronista de comienzos de los años setenta, cuando se registró una participación parlamentaria femenina considerable para los parámetros de la época. 
candidatos a ser ocupados por mujeres debían asegurarles posibilidades ciertas de resultar electas. En ese sentido, se especificó con relativa claridad el sistema de intercalación de los postulantes según su sexo y, adicionalmente, la norma determinó que las listas que no cumplieran con estos requisitos no serían oficializadas. A pesar de las posibilidades de fácil comprensión de estos dispositivos, inicialmente la mayoría de los partidos políticos demostró dificultades para interpretar correctamente la norma. Ante este hecho, los recursos legales que presentaron mujeres a lo largo de todo el país condujeron a la promulgación de un segundo decreto reglamentario en el año 2000 que definió con exactitud la ubicación que deben tener las candidatas en las listas electorales, estableciendo una casuística extensa en previsión de cualquier caso que pueda despertar dudas al respecto.

La legislación brasileña carece de esa precisión. Si bien en un marco de listas abiertas no es posible establecer ninguna especificación con relación al emplazamiento de las candidatas en las nóminas partidarias, los apartados dedicados a las cuotas de género sólo hacen referencia a la obligación de reservar porcentajes mínimos y máximos de candidaturas para cada sexo que, de no alcanzarse, simplemente no pueden completarse con candidaturas del otro sexo. Al mismo tiempo, la ley autoriza a los partidos a postular hasta un $150 \%$ de candidatos respecto del número de escaños por los cuales se compite sin obligarlos a presentar ese porcentaje máximo. En la práctica, este arreglo se presta para neutralizar aún más los efectos de la ya escueta norma.

El decreto reglamentario de la Ley de Cupo Femenino de Argentina, al disponer la intervención de la justicia electoral en los casos de incumplimiento de la norma, define explícitamente un sistema de sanciones. Además, otorga a todos los habitantes de un distrito electoral el derecho de impugnar una lista que consideren violatoria de la ley. La legislación brasileña, por su parte, no incluye ningún sistema de sanciones, dejando de esta manera un vacío legal que permitió al conjunto de los partidos políticos no respetar las cuotas mínimas en las elecciones celebradas en 1998 y 2002.

A la luz del análisis y de la comparación de ambas experiencias nacionales, es posible apreciar que, en el marco de un sistema electoral favorable, la precisión de la provisión de cuotas y la definición clara de las posiciones que deben ocupar las candidatas en las listas partidarias, sumadas a la existencia de sanciones para casos de incumplimiento y a la vigilancia por su correcta aplicación, gravitaron de modo decisivo sobre el éxito del cupo femenino en la Argentina. En contrapartida, la ausencia de estas condiciones en el caso brasileño permite comprender el desempeño más incierto de las cuotas en ese país. 


\section{A modo de reflexión final: el desafío de ampliar el acceso femenino al desempeño de cargos representativos}

Sobre la base de lo expuesto, es posible formular algunas reflexiones que surgen como enseñanzas de la experiencia comparada y que podrían orientar las estrategias encaminadas a incrementar la presencia de mujeres en las instituciones representativas en otras realidades nacionales.

En aquellos países cuyos sistemas electorales presentan condiciones favorables para la aplicación exitosa de cuotas femeninas o de género, es importante que los interesados en promoverlas establezcan alianzas y se movilicen en aras de lograr que las normas sean formuladas con la mayor precisión posible y cuenten con adecuados sistemas de sanciones. Las iniciativas orientadas a vigilar su cumplimiento y, en caso contrario, a velar por su correcta aplicación, también son un factor de suma relevancia. Además, tomando en consideración que aunque estén concebidas como un piso mínimo de participación femenina, las cuotas suelen tender a transformarse en "techos", parecería conveniente reflexionar sobre los porcentajes adecuados a la hora de formular propuestas y de entablar negociaciones.

En países cuyos sistemas electorales son menos favorables para la implementación de cuotas, éstas deberían ser acompañadas por otras medidas complementarias -tales como la introducción de acciones de discriminación positiva en los órganos de dirección de los partidos políticos o respecto de la distribución equitativa de los recursos de campañas- capaces de contrarrestar esas dificultades. El caso de Brasil revela que, en ausencia de tales medidas, la sanción de leyes de cuotas puede no ser suficiente para incrementar las posibilidades de ingreso de mujeres y de otros nuevos actores al campo de la competencia política.

Finalmente, en un escenario en el cual frecuentemente se cuestiona la calidad de la democracia y de las instituciones representativas, sería provechoso prestar suma atención a los debates sobre reforma política que prevén modificaciones en la legislación electoral, para así evaluar anticipadamente sus posibles impactos respecto de las chances de elección de mujeres.

\section{Bibliografía}

Abal Medina, Juan Manuel; Suárez Cao, Julieta; Nejamkis, Facundo. 2003. "Reglas similares, resultados distintos: las instituciones políticas argentinas y brasileñas en perspectiva comparada". En Carlos Álvarez (comp.), La Argentina de Kirchner y el Brasil de Lula. Buenos Aires: CEPES, CEDEC, Prometeo, 241-268.

Allegrone, Norma. 2002. Ley de Cupo Femenino: su aplicación e interpretación en la República Argentina. Buenos Aires: Fundación para el Desarrollo en Igualdad FUNDAI - Fundación Friedrich Ebert. 
Araújo, Clara. 2003. “Las cuotas para mujeres en el sistema legislativo brasileño". En La aplicación de las cuotas: experiencias latinoamericanas. Informe de taller. Lima: International IDEA, 78-94.

1999. Cidadania incompleta: O impacto da lei de cotas sobre a representação política das mulheres no Brasil. Río de Janeiro: Instituto de Filosofia e Ciências Sociais/UFRJ, Programa de Pós-Graduação em Sociologia e Antropología, Tese de Doutorado.

Archenti, Nélida. 2002. "Los caminos de la inclusión política, acciones afirmativas de género". En Silvia Vázquez (comp.). Hombres públicos, mujeres públicas. Buenos Aires: Fundación Friedrich Ebert y Fundación Karakachof, 25-58.

2000. "Representación, Ley de Cuotas y Sistemas Electorales". Revista Postdata (6): 171-194.

Barrancos, Dora. 2002. Inclusión/Exclusión. Historia con Mujeres. Buenos Aires: Fondo de Cultura Económica.

Barreiro, Line; López, Óscar; Soto, Clyde; Soto, Lilian. 2004. Sistemas electorales y representación femenina en América Latina. Santiago de Chile: Proyecto CEPAL/ DAW, Serie Mujer y Desarrollo No 54 .

Blay, Eva. 2002. "Mulher e Igualdade: cidadania e gênero". Socialdemocracia Brasileira 1 (2): 58-63.

Calvo, Ernesto; Escolar, Marcelo. 2005. La nueva política de partidos en la Argentina: Crisis política, realineamientos partidarios y reforma electoral. Buenos Aires: Prometeo.

Consejo Nacional de la Mujer. 1994. Informe nacional: Situación de la mujer en la última década en la República Argentina. Buenos Aires: Centro Nacional de Coordinación Preparatoria de la IV Conferencia Mundial de la Mujer y CMN.

Dahlerup, Drude. 2004. "Estudios comparativos sobre las cuotas de género". En La aplicación de las cuotas: experiencias latinoamericanas. Informe de taller. Lima: International IDEA, 11-21.

Dahlerup, Drude; Freidenvall, Lenita. 2003. “Quotas as a 'Fast Track' to Equal Political Representation for Women". Paper presented at the APSA Annual Meeting in Philadelphia, August 28 to 31.

Deleis, Mónica, et al. 2001. Mujeres de la política argentina. Buenos Aires: Aguilar.

Gray, Tricia. 2003. “Electoral Gender Quotas: Lessons from Argentina and Chile". Bulletin of Latin American Research 22 (1): 52-78.

Htun, Mala. 2003. “Dimensiones de la inclusión y exclusión política en Brasil: Género y raza". Washington DC: Banco Interamericano de Desarrollo.

Htun, Mala; Jones, Mark P. 2002. "Engendering the right to participate in decision-making: Electoral quotas and women's leadership in Latin America". En Craske, Nikki y Molineux, Maxine (eds.) Gender and the Politics of Rights and Democracy in Latin America. New York: Palgrave, 32-56.

Jones, Mark P.; Navia, Patricio. 1999. "Assessing the Effectiveness of Gender Quotas in Open-List Proportional Representation Electoral Systems". Social Science Quarterly 80 (2): 341-355. 
Lázaro, Alejandra; Fraquelli, Ileana. 2003. "Ley de Cupo: ¿Avance Legislativo o Judicial?". En Archenti, Nélida (comp.), Estrategias Politicas de Género: Reformas institucionales, identidad y acción colectiva. Buenos Aires: Instituto Gino Germani de la Facultad de Ciencias Sociales de la Universidad de Buenos Aires.

Lovenduski, Joni; Norris, Pippa. 1996. Women and Politics. Oxford: Oxford University Press.

Macaulay, Fiona. 2003. "Sexual politics, party politics: the PT government's policies on gender equity and equality". Working Paper Number CBS-46-03, Oxford: University of Oxford, Centre for Brazilian Studies.

Marx, Jutta. 1992. Mujeres y Partidos Políticos. De una masiva participación a una escasa representación. Un estudio de caso. Buenos Aires: Legasa.

Matland, Richard. 2004. "El proceso de representación y reclutamiento legislativo de las mujeres". En Mujer, Partidos Políticos y Reforma Electoral. Lima: Internacional IDEA, 13-30.

Miguel, Luis Felipe. 2004. "Participação Eleitoral e gênero no Brasil: As cotas para mulheres e seu impacto". II Congresso da Asociación Latinoanericana de Ciencia Política (ALACIP). México: outubro.

Miguel, Sonia Malheiros. 2000. A política de cotas por sexo. Um estudo das primeiras experiências no Legislativo brasileiro. Brasilia: CFEMEA - Centro de Estudos e Assesoria.

Nohlen, Dieter. 1998. Sistemas electorales y partidos políticos. México: Fondo de Cultura Económica.

Peschard, Jacqueline. 2003. “El sistema de cuotas en América Latina: Panorama general". En La aplicación de cuotas: experiencias latinoamericanas. Informe de Taller. Lima: Internacional IDEA, 22-31.

Reynolds, Andrew. 1999. "Women in the Legislaturas and Excecutives of the World: Knocking at the Highest Glass Ceiling". World Politics 51 (4): 547-572.

Rule, Wilma. 1994. "Parliament of, by, and for the People: Except for Women?". En Wilma Rule and Joseph Zimmerman (eds.). Electoral Systems in Comparative Perspective: Their Impact on Women and Minorities. Wesport, Connecticut and London: Greenwood.

Tula, María Inés. 2002. “La Ley de Cupos en la Argentina: la participación de las mujeres en los órganos representativos de gobierno". En Silvia Vázquez (comp.), Hombres públicos, mujeres públicas. Buenos Aires: Fundación Friedrich Ebert y Fundación Karakachof, 59-90. 
- 